\title{
Towers of isospectral manifolds
}

\author{
Manlio Bordoni, Verita Marino*
}

July 2, 2007

\begin{abstract}
Given two isospectral not isometric manifolds, we construct a new couple of such manifolds as the total spaces of two Riemannian submersions with totally geodesic fibers isometric to the given ones and of basis any other given manifold. By iteration, we obtain families of isospectral not isometric manifolds.
\end{abstract}

\section{Introduction}

We shall assume, in this paper, that all the manifolds are connected, compact and boundaryless. The spectrum of a Riemannian manifold $(M, g)$ is the sequence of the eigenvalues of the Laplace-Beltrami operator $\Delta_{M}=\Delta(M, g)$ acting on $C^{\infty}$ functions defined on $M$ :

$$
\operatorname{Spec}\left(\Delta_{M}\right)=\left\{0=\lambda_{0}<\lambda_{1} \leq \lambda_{2} \leq \cdots \nearrow+\infty\right\}
$$

each eigenvalue is repeated according to its multiplicity, which is finite.

From the definition itself, it follows that two isometric manifolds are also isospectral, but the converse is not true, that is to say there exist isospectral but not isometric manifolds. The first example of such manifolds was given in 1964 by J. Milnor [9] with two 16-dimensional flat tori. After him, several examples was produced by many mathematicians. A fundamental contribution in the isospectrality problem is due to T. Sunada in his famous paper [12] in 1985. Subsequently, the problem was: does isospectrality imply at least local isometry? Also the answer to this question is negative, the first counterexamples in this sense was given by C. S. Gordon, D. Webb and S. Wolpert in 1992,[6]. For a survey on isospectrality, see for instance C. S. Gordon, $[5]$.

${ }^{*}$ Work supported by M.U.R.S.T. of Italy 
Inspirated by the paper [3] of G. Besson and the first author, we illustrate a general method to build up, starting from two isospectral not isometric manifolds, new couples of such manifolds, obtained as total spaces of Riemannian submersions over any basis manifold, with totally geodesic fibers isometric to the two manifolds considered at the beginning. The iteration of this process allows to build up, on any basis manifold, a couple of towers of isospectral not isometric manifolds of higer and higer dimensions: this justify the title of the paper.

In section 2, the preliminaries are presented and it is stated the main theorem (2.1) to construct the first couple of isospecral not isometric manifolds; the proof of this theorem is performed in section 3 . We end by illustrating how to build up the towers and by showing that Sunada's examples are suitable for this construction.

\section{The main theorem}

Let $(B, j),\left(F, g_{F}\right)$ be two Riemannian manifolds of dimensions $n$ and $r$ respectively, and let $G$ be the (compact) Lie group of the isometries of $\left(F, g_{F}\right)$. Consider the fibration $\pi$ associated to the principal bundle $p$ : $P \longrightarrow B$ of structural group $G$ on the basis $B$ with fibers diffeomorphic to $F$ :

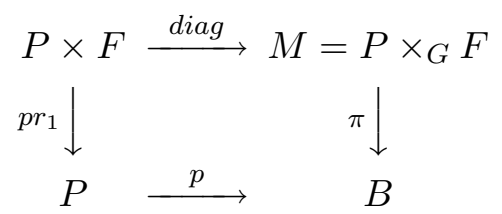

where $p r_{1}$ is the projection on the first factor, and diag is the diagonal action of the group $G$ on the product $P \times F$. Recall that $G$ acts freely without fixed points on the right on $P$ and $B=P / G$. Moreover $G$ acts freely without fixed points on $P \times F$ by the diagonal action. The quotient of $P \times F$ by diag is a $(n+r)$-dimensional manifold, denoted $P \times_{G} F$; in the sequel we shall write briefly $M$ for $P \times_{G} F$. It is well known that the fibration $\pi: M \longrightarrow B$ is a submersion with totally geodesic fibers, see S. Kobayashi and K. Nomizu [8], vol. I p. 54 and B. O'Neill [10].

Choose a suitable biinvariant metric on $G$, this gives a Riemannian metric on $P$ which makes the projection map $p$ a Riemannian submersion with totally geodesic fibers isometric to $F$, see J. Cheeger[4]. The product metric on $P \times F$ pass to the quotient and it gives a Riemannian metric $g$ on $M=P \times_{G} F$. In this case all the fibers $F_{x}=\pi^{-1}(x), x \in B$, are regular $r$-dimensional submanifolds of $M$, and they, endowed with the metric $g_{x}$ induced by $g$, are isometric to 
the model fiber $\left(F, g_{F}\right)$, see B. O'Neill [10], R. Hermann [7].

With respect to the Laplacians, L. Bérard Bergery and J. P. Bourguignon introduce in [1] a decomposition of $\Delta_{P}$ as a sum of two operators:

$$
\Delta_{P}=\Delta_{P}^{h}+\Delta_{P}^{v}
$$

the so called horizontal and vertical Laplacians, corresponding to the natural decomposition of the metric. Namely, the vertical Laplacian is defined to be the operator

$$
\left(\Delta_{P}^{v} f\right)(x)=\left(\Delta_{P_{x}}\left(f \uparrow_{P_{x}}\right)\right)(x)
$$

where $P_{x}$ is the fiber of $p$ through $x \in B$; in other words, $\Delta_{P}^{v}$ is the Laplacian of the fibers of $p$. The operator

$$
\Delta_{P}^{h}=\Delta_{P}-\Delta_{P}^{v}
$$

is the horizontal Laplacian. In general, these two operators are not elliptic, however they have discrete spectra and their eigenvalues have finite multiplicity. When the fibers are totally geodesic, as in the case under consideration, vertical and horizontal Laplacians commute then the eigenvalues of $\Delta_{P}$ are (not all) the sums of the eigenvalues of each of them. The eigenvalues of $\Delta_{P}^{h}$ are in substance the ones of $\Delta_{B}$, and all of them appear in the sums; we shall see later that the eigenvalues of the vertical Laplacian do not take part in our problem.

Consider now two Riemannian manifolds $\left(F_{1}, g_{F_{1}}\right),\left(F_{2}, g_{F_{2}}\right)$ with isometry groups $G_{1}$ and $G_{2}$ respectively. The previous construction gives two Riemannian submersions

$$
\pi_{1}:\left(M_{1}, g_{1}\right) \longrightarrow(B, j) \text { and } \pi_{2}:\left(M_{2}, g_{2}\right) \longrightarrow(B, j),
$$

with totally geodesic fibers isometric to $\left(F_{1}, g_{F_{1}}\right)$ and $\left(F_{2}, g_{F_{2}}\right)$ respectively:
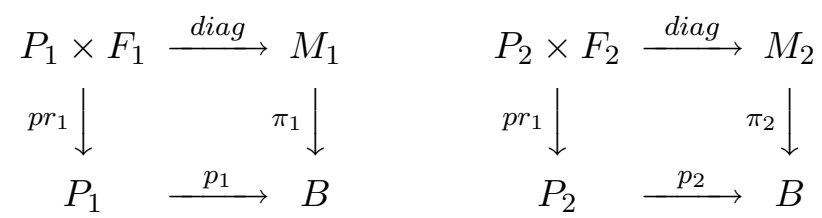

The main result of this paper is:

Theorem 2.1 Let $(B, j)$ be any Riemannian manifold, and let $\left(F_{1}, g_{F_{1}}\right)$, $\left(F_{2}, g_{F_{2}}\right)$ be two isospectral manifolds, and assume that their isometry groups $G_{1}, G_{2}$ are isomorphic as abstract groups. Then the manifolds $M_{1}=P_{1} \times_{G_{1}} F_{1}$ and $M_{2}=P_{2} \times_{G_{2}} F_{2}$, endowed with the induced metrics $g_{1}$ and $g_{2}$, are also isospectral. 


\section{Proofs}

In the proof of 2.1, an important tool is the theory of complex irreducible unitary representations of groups.

Lemma 3.1 Two isomorphic compact Lie Groups $G_{1}, G_{2}$ have the same complex irreducible unitary representations.

Proof. Let $\alpha: G_{1} \longrightarrow G_{2}$ be an isomorphism and let $\rho: G_{1} \longrightarrow$ $G L(S)$ be a complex irreducible unitary representation. For $g_{1} \in G_{1}$ denote $g_{2}=\alpha\left(g_{1}\right)$ and define $\sigma: G_{2} \longrightarrow G L(S)$ by setting

$$
\sigma_{g_{2}}=\sigma\left(g_{2}\right)=\sigma\left(\alpha\left(g_{1}\right)\right):=\rho\left(g_{1}\right) \text { i.e. } \sigma=\rho \circ \alpha^{-1} .
$$

The representation $\sigma$ is irreducible. Indeed, if there exists a non trivial subspace $V$ of $S$ which is $G_{2}$-invariant, one has $\sigma_{g_{2}}(v) \in V \forall v \in V$ and $\forall g_{2} \in G_{2}$. This implies $V \ni \sigma_{g_{2}}(v)=\sigma_{\alpha\left(g_{1}\right)}(v)=\rho_{g_{1}}(v) \forall g_{1} \in G_{1}$ (because $\alpha$ is an isomorphism) and $\forall v \in V$, so $V$ is $G_{1}$-invariant, in contradiction with the assumption that $\rho$ is irreducible. Moreover, $\sigma$ is unitary as

$$
\left\langle\sigma_{g_{2}}(v), \sigma_{g_{2}}(w)\right\rangle=\left\langle\rho_{g_{1}}(v), \rho_{g_{1}}(w)\right\rangle=\langle v, w\rangle
$$

for any $v, w \in S(\langle.,$.$\rangle denotes the inner product in S)$.

Denote $\mathcal{R}$ the set of complex irreducible unitary representations of the groups $G_{1}$ and $G_{2}$ : they are finite dimensional, since the groups are compact. As $G_{1}$ and $G_{2}$ act by isometries on $\left(F_{1}, g_{F_{1}}\right)$ and $\left(F_{2}, g_{F_{2}}\right)$ resp., we can consider the canonical decompositions of the spaces $L^{2}\left(F_{1}\right)$ and $L^{2}\left(F_{2}\right)$ in isotypical components (they are $L^{2}$-orthogonal)

$$
L^{2}\left(F_{1}\right)=\oplus_{\rho \in \mathcal{R}} L_{\rho}^{2}\left(F_{1}\right) \quad, \quad L^{2}\left(F_{2}\right)=\oplus_{\rho \in \mathcal{R}} L_{\rho}^{2}\left(F_{2}\right) .
$$

We have also another decomposition of these two spaces. Let us call $\mu_{1}<\mu_{2}<\ldots$ the eigenvalues of the Laplace-Beltrami operators $\Delta_{F_{1}}$ and $\Delta_{F_{2}}$ : as the manifolds $F_{1}$ and $F_{2}$ are isospectral, $\Delta_{F_{1}}$ and $\Delta_{F_{2}}$ have the same eigenvalues and each of them has the same multiplicity. Thus, the eigenspaces $E_{F_{1}}\left(\mu_{i}\right)$ and $E_{F_{2}}\left(\mu_{i}\right)$ have the same (finite) dimension and they are consequentely isomorphic. The spaces $L^{2}\left(F_{j}\right)$ have also the following $L^{2}$-orthogonal decompositions:

$$
L^{2}\left(F_{1}\right)=\oplus_{i} E_{F_{1}}\left(\mu_{i}\right) \quad, \quad L^{2}\left(F_{2}\right)=\oplus_{i} E_{F_{2}}\left(\mu_{i}\right) .
$$

As $G_{1}$ and $G_{2}$ act by isometries on $\left(F_{1}, g_{F_{1}}\right)$ and $\left(F_{2}, g_{F_{2}}\right)$ resp., we have the similar decompositions in isotypical components

$$
E_{F_{1}}\left(\mu_{i}\right)=\oplus_{\rho \in \mathcal{R}} E_{F_{1}}^{\rho}\left(\mu_{i}\right) \quad, \quad E_{F_{2}}\left(\mu_{i}\right)=\oplus_{\rho \in \mathcal{R}} E_{F_{2}}^{\rho}\left(\mu_{i}\right) .
$$


As $E_{F_{1}}\left(\mu_{i}\right)$ and $E_{F_{2}}\left(\mu_{i}\right)$ are isomorphic, these two decompositions coincide. Notice that for any fixed $\rho \in \mathcal{R}$, one has

$$
E_{F_{j}}^{\rho}\left(\mu_{i}\right)=E_{F_{j}}\left(\mu_{i}\right) \cap L_{\rho}^{2}\left(F_{j}\right) \quad j=1,2,
$$

this means that not all the representations $\rho$ appear in a fixed eigenspace and that the same $\rho$ may appear in different eigenspaces. The number of times in which a fixed $\rho$ appears in the eigenspaces $E_{F_{1}}\left(\mu_{i}\right)$ and $E_{F_{2}}\left(\mu_{i}\right)$ is the same.

We consider also the decomposition in isotypical components of $L^{2}\left(P_{j}\right), j=1,2$ :

$$
L^{2}\left(P_{1}\right)=\oplus_{\rho \in \mathcal{R}} L_{\rho}^{2}\left(P_{1}\right) \quad, \quad L^{2}\left(P_{2}\right)=\oplus_{\rho \in \mathcal{R}} L_{\rho}^{2}\left(P_{2}\right) .
$$

For a fixed $d$-dimensional representation $\rho \in \mathcal{R}$, consider two isomorphic irreducible non trivial $d$-subspaces $V_{1}$ and $V_{2}$ of $L_{\rho}^{2}\left(P_{1}\right)$ and $L_{\rho}^{2}\left(P_{2}\right)$ which are $G_{1^{-}}$, resp. $G_{2^{-}}$, invariant. For the same $\rho$, consider also two isomorphic irreducible non trivial $d$-subspaces $W_{1}$ and $W_{2}$ of $L_{\rho}^{2}\left(F_{1}\right)$ and $L_{\rho}^{2}\left(F_{2}\right)$ which are $G_{1^{-}}$, resp. $G_{2^{-}}$, invariant. By Schur's lemma, there exists a unique (up to a complex number of modulus 1) equivariant isometry $\alpha_{V_{j} W_{j}}$ between $V_{j}$ and $W_{j}, j=1,2$.

Definition 3.2 Let $\Psi^{j}=\left(\psi_{1}^{j}, \ldots, \psi_{d}^{j}\right)$ be an orthonormal basis of $V_{j}$ and let $\Phi^{j}=\left(\varphi_{1}^{j}, \ldots, \varphi_{d}^{j}\right)$, with $\varphi_{i}^{j}=\alpha_{V_{j} W_{j}}\left(\psi_{i}^{j}\right)$, be the corresponding orthonormal basis of $W_{j}$. Define the scalar product

$$
\left\langle\Psi^{j}, \Phi^{j}\right\rangle=\frac{1}{d} \sum_{i=1}^{d} \psi_{i}^{j} \bar{\phi}_{i}^{j}
$$

(the functions $\psi_{i}^{j}$ and $\phi_{i}^{j}$ are lifted on the product $P_{j} \times F_{j}$ by the canonical projections).

It is easy to verify that the function $\left\langle\Psi^{j}, \Phi^{j}\right\rangle, j=1,2$, is invariant under the action of $G_{j}$ on $P_{j} \times F_{j}$, and that it does not depend on the choice of the basis $\Psi^{j}$, thus it pass to the quotient $M_{j}=P_{j} \times_{G_{j}} F_{j}$; in the sequel we shall consider $\left\langle\Psi^{j}, \Phi^{j}\right\rangle$ as a function on $M_{j}$.

Theorem 3.3 The family of the functions $\left\langle\Psi^{j}, \Phi^{j}\right\rangle$ defined by (8) associated to all the possible pairs $\left(V_{j}, W_{j}\right)$ is a Hilbertian basis of the space of the $L^{2}$-functions on $P_{j} \times F_{j}$ invariant under the action of $G_{j}$, i.e. the space $L^{2}\left(M_{j}\right)$.

Proof. Let $L_{G_{j}}^{2}\left(P_{j} \times F_{j}\right)$ denote the space of $L^{2}$-functions on $P_{j} \times F_{j}$ invariant under the action of $G_{j}$, and consider the decomposition (cf. (3), (7))

$$
L^{2}\left(P_{j} \times F_{j}\right)=L^{2}\left(P_{j}\right) \otimes L^{2}\left(F_{j}\right)=\sum_{\rho, \rho^{\prime} \in \mathcal{R}} L_{\rho}^{2}\left(P_{j}\right) \otimes L_{\rho^{\prime}}^{2}\left(F_{j}\right) .
$$


Then for $\eta \in L_{G_{j}}^{2}\left(P_{j} \times F_{j}\right)$ the classical relations on the characters (see for instance [11], p. 28,48) give that the orthogonal projection of $\eta$ on $L_{\rho}^{2}\left(P_{j}\right) \otimes L_{\rho^{\prime}}^{2}\left(F_{j}\right)$ is equal to zero if $\rho^{\prime} \neq \bar{\rho}$, that is to say

$$
L_{G_{j}}^{2}\left(P_{j} \times F_{j}\right)=\sum_{\rho \in \mathcal{R}} L_{\rho}^{2}\left(P_{j}\right) \otimes L_{\bar{\rho}}^{2}\left(F_{j}\right) .
$$

Moreover, it is clear by computing $L^{2}$-scalar products that for different choices of the couples $\left(V_{j}, W_{j}\right)$ the corresponding functions $\left\langle\Psi^{j}, \Phi^{j}\right\rangle$ are $L^{2}$-orthogonal.

$V_{j}$ and $W_{j}$ are in fact included in some eigenspaces of $\Delta_{P_{j}}$ and $\Delta_{F_{j}}$ resp., related to certain eigenvalues $\alpha^{j}+\beta^{j} \in \operatorname{Spec}\left(\Delta_{P_{j}}\right)$ with $\alpha^{j} \in$ $\operatorname{Spec}\left(\Delta_{P_{j}}^{h}\right)$ and $\beta^{j} \in \operatorname{Spec}\left(\Delta_{P_{j}}^{v}\right)$, and $\mu^{j} \in \operatorname{Spec}\left(\Delta_{F_{j}}\right)$ respectively:

$$
\Delta_{P_{j}} \psi^{j}=\Delta_{P_{j}}^{h} \psi^{j}+\Delta_{P_{j}}^{v} \psi^{j}=\alpha^{j} \psi^{j}+\beta^{j} \psi^{j} \quad, \quad \Delta_{F_{j}} \varphi^{j}=\mu^{j} \varphi^{j} .
$$

Theorem 3.4 The family of the functions $\left\langle\Psi^{j}, \Phi^{j}\right\rangle$ is a Hilbertian basis of $L^{2}\left(M_{j}\right)$ consisting in eigenfunctions of $\Delta_{M_{j}}$. The eigenvalue corresponding to the eigenfunction $\left\langle\Psi^{j}, \Phi^{j}\right\rangle$ is $\alpha^{j}+\mu^{j}$.

For the proof, see [3]; in this paper it is also given the following explicit formula for the multiplicity of a given eigenvalue $\nu^{j}$ of $\operatorname{Spec}\left(\Delta_{M_{j}}\right)$ :

$$
\operatorname{mult}\left(\nu^{j}\right)=\sum_{\rho \in \mathcal{R}} \sum_{\alpha^{j}+\mu^{j}=\nu^{j}} m_{\alpha^{j}}(\rho) m_{\mu^{j}}(\bar{\rho})
$$

with $\alpha^{j} \in \operatorname{Spec}\left(\Delta_{P_{j}}^{h}\right), \mu^{j} \in \operatorname{Spec}\left(\Delta_{F_{j}}\right)$ and where $m_{\alpha^{j}}(\rho)$, resp. $m_{\mu^{j}}(\bar{\rho})$, is the multiplicity of the representation $\rho$, resp. $\bar{\rho}$, in the eigenspace of $\Delta_{P_{j}}^{h}$, resp. $\Delta_{F_{j}}$, related to the eigenvalue $\alpha^{j}$, resp. $\mu^{j}$. It is clearly finite.

Notice that not all sums $\alpha^{j}+\mu^{j}$ are eigenvalues of $\Delta_{M_{j}}$ : if $\alpha^{j}$ correspond to a representation $\rho, \mu^{j}$ must correspond to $\bar{\rho}$, and all unitary irreducible representations of $G_{j}$ appear in $L^{2}\left(P_{j}\right)$, but not all do in $L^{2}\left(F_{j}\right)$. Notice also that the vertical Laplacian $\Delta_{P_{j}}^{v}$ does not give any contribution in $\operatorname{Spec}\left(\Delta_{M_{j}}\right)$.

\section{Conclusion}

Theorem 3.4 and formula (10) show that, when the manifolds $F_{1}, F_{2}$ are isospectral and not isometric, the manifolds $M_{1}, M_{2}$ defined in section 2 have the same eigenvalues, each of them with the same multiplicity, that is they are isospectral and not isometric too. The metrics 
$g_{1}, g_{2}$ defined on $M_{1}, M_{2}$ and such that the projections $\pi_{1}, \pi_{2}$ on $B$ are Riemannian submersions with totally geodesic fibers isometric to $F_{1}, F_{2}$ respectively, decompose each in horizontal and vertical part. The horizontal parts are the same, because both are isometric to the metric of the basis $B$, while the vertical parts are the metrics of the fibers. Therefore the isometry groups of $M_{1}$ and $M_{2}$ are isomorphic because they must respect the decomposition in horizontal and vertical parts (these are orthogonal), and they come from the group of the isometries of $B$ and from the groups $G_{1}$ and $G_{2}$ of the isometries of $F_{1}$ and $F_{2}$, which are isomorphic. Consequently, the process of construction of isospectral manifolds can be repeated starting from $B$ and $M_{1}, M_{2}$. By iteration, we get so two families of isospectral manifolds fibered on $B$, in each of them the fibers are the manifolds obtained in the previous step: these families are the "towers".

Example 4.1 Consider now the isospectral manifolds of Sunada [12]. Let us recall that, given a 4-dimensional manifold $M_{0}$ with fundamental group $G$ and whose universal covering is denoted $p: M \longrightarrow M_{0}$, Sunada builds up the intermediate coverings $p_{1}: F_{1}=M / H_{1} \longrightarrow M_{0}$ and $p_{2}: F_{2}=M / H_{2} \longrightarrow M_{0}$ having as fundamentals groups two non conjugate subgroups $H_{1}, H_{2}$ of $G$, and he shows that, when $H_{1}$ and $H_{2}$ are not isomorphic, then the manifolds $\left(F_{1}, p_{1}^{*} g_{0}\right)$ and $\left(F_{2}, p_{2}^{*} g_{0}\right)$ are isospectral but not isometric for any metric $g_{0}$ on $M_{0}$. However, also in the case in which $H_{1}$ and $H_{2}$ are isomorphic as abstract groups, such manifolds are isospectral and not isometric for some metric $g_{0}$ on $M_{0}$. For instance, this happens when the isometry group of the universal covering space $M$ of $M_{0}$, endowed with the metric $p^{*} g_{0}$ coincides with the covering transformation group $G$. In this case, the isometry groups $G_{1}=G / H_{1}$ of $\left(F_{1}, p_{1}^{*} g_{0}\right)$ and $G_{2}=G / H_{2}$ of $\left(F_{2}, p_{2}^{*} g_{0}\right)$ are isomorphic as abstract groups since $H_{1}$ and $H_{2}$ are. Then, we can use the manifolds $F_{1}, F_{2}$ to apply our construction.

\section{References}

[1] Bérard Bergery, L.; Bourguignon, J.P. :Laplacians and Riemannian submersions with totally geodesic fibers, Illinois Journal of Math., 26, n.2 (1982),181-200

[2] Besson,G.: A Kato type inequality for Riemannian submersions with totally geodesic fibers, Annals of Global Analysis and Geometry Vol. 4, n. 3 (1986), 273-289

[3] Besson, G.; Bordoni, M.: On the spectrum of Riemannian submersions with totally geodesic fibers, Rend. Mat. Acc. Lincei s. 9, v. 1 (1990), 335-340 
[4] Cheeger,J. : Some examples of manifolds of non negative curvature, J. Diff. Geom. 8 (1972), 623-628

[5] Gordon, C. S. : Survey of isospectral manifolds, Dillen, Franki J. E. (ed) et al., Handbook of differential geometry. Volume I. Amsterdam: North-Holland (2000), 747-778

[6] Gordon, C. S.; Webb, D.; Wolpert, S. Isospectral plane domains and surfaces via Riemannian orbifolds, Invent. Math. 110 (1992), no. $1,1-22$.

[7] Hermann, R.: A sufficient condition that a mapping of Riemannian manifolds be e fibre bundle, Proc. Amer. Math. Soc., vol. II (1960), 236-242

[8] Kobayashi, S.; Nomizu, K.: Foundations of differential Geometry, Vol. I, Wiley Interscience, New York-London 1963

[9] Milnor, J.: Eigenvalues of the Laplace operators on certain manifolds, Proc. Nat. Acad. Sci. USA 51 (1964), 542

[10] O'Neill, B. : The fundamental equations of a submersion, Michigan Math. J. 13 (1966), 459-469

[11] Serre, J. P. : Representations linéaires des groupes finis, Hermann, Paris 1971

[12] Sunada, T. : Riemannian coverings and isospectral manifolds, Ann. of Math. 121 (1985), 169-186

Manlio Bordoni

Università degli Studi di Roma "La Sapienza"

Dipartimento Me.Mo.Mat.

Via A. Scarpa 16

00128 Roma - Italia

bordoni@dmmm.uniroma1.it

Verita Marino

Università della Calabria

Dipartimento di Matematica

Ponte P. Bucci, Cubo 31B

87036 Arcavacata di Rende (Cs) - Italia

marino@unical.it 